Measuring Change in Cannabis Use

\title{
Measuring Change in Cannabis Use
}

Simon J. Adamson (Ph.D) $)^{1^{*}}$

Frances J. Kay-Lambkin (Ph.D) ${ }^{2}$

Amanda L. Baker (Ph.D) $)^{3}$

Chris M. A. Frampton (Ph.D) ${ }^{1}$

Terry J. Lewin (B.Com.(Psych)Hons.) ${ }^{3}$

J. Doug Sellman (Ph.D) ${ }^{1}$

1. National Addiction Centre, Department of Psychological Medicine, Christchurch School of Medicine and Health Sciences, University of Otago, PO Box 4345, Christchurch, New Zealand.

2. National Drug and Alcohol Research Centre, University of New South Wales, Sydney NSW 2052, Australia.

3. Priority Research Centre for Translational Science and Mental Health, University of Newcastle, PO Box 833, Newcastle, NSW, Australia.

* Corresponding author 


\begin{abstract}
We examined the ability of the Cannabis User Disorders Identification Test - Revised (CUDIT-R) to detect change in a treatment sample, including correlation with changes in other clinically relevant areas of functioning, and to determine reliable and clinically significant change thresholds. 133 cannabis-using patients taking part in a treatment trial for concurrent substance use and mood disorder were administered the 8-item CUDIT-R at baseline, 6 and 12 months, in addition to assessment of current cannabis use disorder, mood, alcohol use, motivation and employment status. Significant reductions in CUDIT-R scores were observed and were correlated with change in cannabis diagnosis, and improvement in mood. Higher motivation at baseline predicted greater reduction in CUDIT-R score. Reliable change was identified as occurring when CUDIT-R score changed by two or more, while clinically significant change, benchmarked against an increase or decrease of one DSM-IV cannabis dependence symptom, was equated to a CUDIT-R score changing by three or more points.
\end{abstract}

Keywords: Cannabis, Measurement, Outcome, Improvement 


\section{INTRODUCTION}

Cannabis is the most commonly used illicit drug in the western world (United Nations Office on Drugs and Crime, 2010). Heavy regular cannabis use is associated with a number of negative outcomes, including poor mental health (Hall \& Degenhardt, 2008; Patton et al., 2002), impaired cognitive functioning (Bolla et al., 2002; Meier et al., 2012; Pope et al., 2001), reduced educational attainment (Horwood et al., 2010), road accidents (Fergusson et al., 2008; Ramaekers et al., 2004), poor lung functioning (Tashkin, 1999), and contentiously, as a gateway to other substance use (Fergusson et al., 2006).

Although a range of treatments have been shown to be effective for cannabis use (McRae et al 2003; Nordstrom \& Levin 2007) a better understanding of what constitutes effective treatment and how best to measure change, is needed. There are a range of instruments designed to measure cannabis use severity in general (Cannabis Use Disorders Identification Test - Revised (CUDIT-R), Adamson et al 2010; Cannabis Use Problems Identification Test (CUPIT), Bashford et al., 2010) or specific symptoms or areas of functioning (Marijuana Craving Questionnaire (MCQ), Heishman et al., 2001; Cannabis Problems Questionnaire (CPQ; Copeland et al., 2001). Additionally, there are more generic tools such as the Alcohol, Smoking and Substance Involvement Screening Test (ASSIST; WHO ASSIST Working Group, 2002) and Severity of Dependence Scale (SDS; Gossop et al., 1995) used to measure cannabis use as one of multiple substances. None of these have to date been examined for their ability to measure change in cannabis use severity, an important function in clinical settings or for longitudinal population studies.

The Cannabis Use Disorders Identification Test - Revised (CUDIT-R; Adamson et al., 2010) was originally developed as the CUDIT (Adamson \& Sellman 2003). The original scale was a simple modification of the AUDIT. At the time of publication the authors identified shortcomings with certain items, a concern reinforced by other studies (Annaheim et al., 2008, 2010; Piotnek et al., 
2008) Subsequent refinement led to the development of the briefer 8-item CUDIT-R (Adamson et al., 2010). Possible scores range from 0 to 32 and items cover the domains of consumption, cannabis problems, dependence and psychological features. The CUDIT-R was found to comprise a single factor, had high test-retest reliability ( $r=0.871)$, high internal consistency $(\alpha=0.914)$ and discriminant validity (AUC=0.960). A later analysis with a US young adult community sample reported much lower internal consistency $(\alpha=0.66)$ but good construct validity with scores correlated with past month use ( $r=.36)$ (Ramo et al 2012). An Australian adult community sample found the CUDIT-R to be unidemensional, strongly correlated with SDS scores, DSM-V and DSM5 symptoms counts (all $r>$.7) and able to distinguish dependence severity (eg AUC for DSM-5 moderate cannabis use disorder $=0.89$ ) (Bruno et al 2013). Bruno et al noted that the CUDIT-R provided good levels of sensitivity and specificity for a community sample but that these were lower than was the case for clinical samples. The CUDIT-R was strongly correlated with cannabis attentional bias in a sample of cannabis users, while the MCQ was not (Cousijns et al 2013)

This paper presents analyses of follow-up CUDIT-R data in a clinical sample. The aims were to determine: (i) whether CUDIT-R scores change significantly over time in a clinical treatment sample; (ii) the extent to which change in CUDIT-R scores parallels other changes in clinically relevant areas of functioning; (iii) the amount of change required to constitute reliable change; and (iv) the degree of change that might be considered clinically significant.

\section{MATERIALS AND METHODS:}

\section{Sample}

The current sample was drawn from a larger study in a clinical trial of cognitive behavioural therapy (CBT) for depression and substance misuse (Kay-Lambkin et al., 2011). Patients, recruited via public advertisements and referral, to the Self-Help for Alcohol/drug use and Depression (SHADE) study met criteria for current depressive symptoms, scored 17 or greater on the Beck 
Depression Inventory II (BDI-II; Beck, 1993) and had concurrent hazardous use of alcohol, cannabis and/or amphetamines. The SHADE study successfully enrolled 274 participants, all of whom provided informed consent to participate. The follow up rate at 6 months was $60.6 \%(n=166)$ and at 12 months, 59.9\% ( $\mathrm{n}=164)$. An earlier study (Kay-Lambkin et al., 2011) found no significant difference between those attending follow-up and those lost to the study on substance use or depression At baseline 136 participants reported using cannabis. Due to some missing data for the CUDIT-R the available sample for analysis was 129. At 6 months CUDIT-R data were available for 76 of these participants and at 12 months for 74 participants. In addition there were 4 participants without baseline CUDIT-R data for whom both 6 and 12 month data were available. A subsample completed a one week test-retest administration of the CUDIT-R following baseline, with 66 complete pairs available for analysis.

\section{Intervention}

Following an initial session, participants in the SHADE trial received nine weekly treatment sessions of either 1) combined cognitive behavioural therapy and motivational interviewing from a therapist or 2) computer delivered with brief therapist assistance at the end of each session, or 3) supportive counselling. Cannabis use outcomes did not differ between conditions (Kay-Lampkin et al., 2011)

\section{Assessment}

In addition to the CUDIT-R, participants were also administered the substance use disorder section of the Structured Clinical Interview for DSMIV (SCID, Research Version; Spitzer et al., 1988), which generated clinician-assessed cannabis use disorder diagnoses for the past 6 months. Mood symptoms were measured using the BDI-II, and alcohol misuse using the Alcohol Use Disorders Identification Test (AUDIT; Saunders et al. 1993). All 4 measures were administered at baseline and both follow-up interviews. Motivation to change cannabis use was measured at baseline using 
Measuring Change in Cannabis Use

the Readiness to Change Questionnaire - Treatment Version (RCQ-TV; Heather et al., 1999). A full copy of the CUDIT-R can be found as an appendix to the online copy of Adamson et al. (2010).

\section{Analysis}

Non-parametric statistics were used to analyse data that were not normally distributed: Wilcoxon test for change in mean score, Spearman's rho for correlation between continuous or ordinal variables, while Pearson's r was used for correlations between normally distributed variables.

To better convey the magnitude of score change participants were categorised into 4 groups based on CUDIT-R scores. Adamson et al (2010) reported that the optimal cut-off for identifying a cannabis use disorder was 13. Mean (SD) CUDIT-R scores were 5.90 (4.52) for those with no diagnosed cannabis use disorder, 13.82 (4.36) for cannabis abuse and 23.95 (6.53) for cannabis dependence. With this information, and the score distributions shown in Figure 1 in mind, the sample was divided into 4 groups based on CUDIT-R score, no use (0) and sub-threshold (1-12), medium (13-20) and high (21-32) problem levels.

Reliable change was deemed to have occurred when an individual score increased or decreased by more than $1.96 \times$ SE of the difference between test scores from time 1 to time 2 (i.e. the reliable change index, Jacobson \& Truax 1991). Linear regression was used to identify the slope (and 95\% confidence interval) for the relationship between change in CUDIT-R score and change in DSM-IV cannabis dependence criteria count as a measure of clinically significant change.

\section{RESULTS}

The sample was 63\% male, with a mean age of 36.3 years (SD 8.9), 24\% were in de facto or married relationships, 57\% were parents and 24\% were in paid employment. Mean age of first cannabis use was 16.4 years (SD 4.8). In the 6 months prior to study intake $34 \%$ had used 
Measuring Change in Cannabis Use

amphetamine type stimulants and $27 \%$ met criteria for amphetamine abuse or dependence, while 89\% had used alcohol and 70\% met criteria for alcohol abuse or dependence. At baseline the mean BDI-II score was 32.64 (SD 8.53) with most (75.7\%) equally divided between moderate and severe depression. Motivation to change cannabis use was highly variable, with classification using the RCQ-TV spread across action (41.7\%), contemplation (34.8\%), and precontemplation (23.5\%).

Baseline CUDIT-R scores ranged from 1 to 32, with a mean of 18.37 (SD 9.89), and were not normally distributed, as displayed in Figure 1. In contrast, CUDIT-R change scores were normally distributed.

Figure 1 about here

Scores at each time point are shown in Table 1. Differences from baseline to each follow-up point are highly significant (Wilcoxon test for change $\mathrm{p}<.001$ for baseline to 6 months and baseline to 12 months) but not for change from 6 to 12 months $(p=.373)$. Whilst a significant minority of the sample became abstinent at follow-up (25\% at 6 months, 32\% at 12 months), mean score reduction on the CUDIT-R was not solely due to these participants ceasing their cannabis use. When abstainers were excluded, the change in score from baseline remains significant at 6 months $(p=.026, n=56)$ and 12 months $(p=.012, n=49)$. The reduction from baseline to 6 months represents an effect size of 0.465 , from baseline to 12 months 0.538 , and from 6 months to 12 months 0.074 .

Table 1 about here

\section{Reliable change}


Reliable change indices (improved, unchanged, deteriorated; Jacobson \& Truax, 1991) were calculated for the sample for the following timepoints: baseline to 6 months, baseline to 12 months, 6 months to 12 months and baseline to 1 -week test-retest. The CUDIT-R asks respondents to answer in relation to their cannabis use over the past 6 months, so almost no change in the underlying construct being measured would be expected to occur after only one week. Table 2 shows variability in scores between baseline and follow up as well as the one week test-retest data. A standard reliable change index, based on the standard error of change for test-retest data of 0.551 , would require change greater than 1.08 points (ie 2 or more) on the CUDIT-R.

\section{Table 2 about here}

Adopting this criterion for reliable change, Table 2 shows that substantially more patients reported improvement rather than deterioration, a ratio of approximately 4:1 at both 6 and 12 months. Change from 6 to 12 months shows only a slightly higher rate of improvement than deterioration, with the largest group unchanged, and a similar pattern found for baseline one-week test-retest. An examination of the scatterplot baseline x 6 months (Figure 2) shows that those who abstained were spread across the range of problem severity as measured at baseline.

Figure 2 about here

As expected, variability of scores was minimal from baseline to one week. Examination of change scores from 6 to 12 months in Table 2 suggests a similar pattern. However, the distributions were quite different, since for the latter contrast almost a quarter (15 out of 64 participants) were abstinent at both time points so score remains unchanged, while for the remaining participants there was greater spread. When the descriptive statistics for change are repeated without this sustained abstinence group, the mean change score from 6 to 12 months was $-0.80(n=49, S D=6.171)$. 


\section{Concurrent validity}

As well as change in score, change in diagnostic category was also evident. As displayed in Table 3, among the 79 participants followed from baseline to 6 months 35 (45.5\%) experienced a reduction in diagnostic severity, 37 (48.1\%) were unchanged and 5 (6.5\%) increased in diagnostic severity. Of 51 participants who were dependent at baseline, 23 no longer met DSM-IV diagnostic criteria for cannabis dependence, close to half by becoming abstinent, with the same proportion still using but not meeting criteria for any diagnosis, while only 3 reduced from dependence to abuse.

\section{Table 3 about here}

Changes in CUDIT-R category (Table 1) and cannabis diagnosis (Table 3) from baseline to 6 months were calculated, with possible scores ranging from -3 (greatest severity at baseline then abstinence at 6 months) to 2 (lowest severity use at baseline then greatest severity at 6 months). Change in CUDIT-R score was strongly correlated with change in diagnostic category from baseline to 6 months (Spearman's rho=0.666, $\mathrm{p}<.001$ ), from baseline to 12 months (Spearman's rho=0.755, $\mathrm{p}<.001$ ) and from 6 to 12 months (Spearman's rho=0.482, $\mathrm{p}<.001$ ).

\section{Convergent validity}

The association between CUDIT-R score change and change in other areas of functioning was examined, as outlined in Table 4. Change in CUDIT-R, BDI-II and AUDIT scores were all normally distributed. Associations with CUDIT-R change scores were therefore undertaken using Pearson's correlation coefficient for these variables. Employment as a binary variable could be represented as a score of -1 (loss of employment), 0 (no change in employment/unemployment status) or 1 (gaining employment). For this variable, associations with CUDIT-R change scores were undertaken using Spearman’s rho. 
Table 4 about here

Improvement in cannabis use as measured by the CUDIT-R was modestly correlated with improvement in mood, as measured by the BDI-II from baseline to both 6 and 12 months and between 6 months and 12 months. No association was found between CUDIT-R and alcohol use as measured by the AUDIT between baseline and either 6 or 12 months, and between 6 and 12 months. Although there was no association between change in CUDIT-R and employment status from baseline to either follow-up, there was a marginally significant association $(\mathrm{p}<.05)$ from 6 to 12 months, with a reduction in CUDIT-R score being associated with reduced employment.

The ability of motivation to predict change in cannabis use was also explored. Baseline RCQ-TV scores predicted greater reduction in CUDIT-R scores from baseline to 6 months $(r=-0.244, B=-$ $0.231 \mathrm{n}=72, \mathrm{p}=0.039$ ) with a trend also present for change in CUDIT-R score from baseline to 12 months $(r=-0.223, B=-0.199, n=68, p=0.068)$.

\section{Clinical significance}

Baseline CUDIT-R score was strongly correlated with DSM-IV dependence criteria symptom count $(r=0.798, p<.001)$. Linear regression revealed that a 1-point difference in number of cannabis dependence criteria corresponded to a 3-point difference in CUDIT-R score $(B=2.937,95 \% \mathrm{CI}$ : 2.485-3.389, $\mathrm{n}=97)$. Change in CUDIT-R score from baseline to 6 months was more modestly correlated with change in dependence criteria count $(\mathrm{r}=0.530, \mathrm{p}=.004)$ with a somewhat smaller degree of change in CUDIT-R score per dependence criterion (B=2.121, 95\%CI: 0.723-3.519, $\mathrm{n}=28$ ). Taking these two analyses together a 3 point difference in CUDIT-R score can be considered as corresponding to a difference of a single cannabis dependence criterion. 


\section{DISCUSSION}

Overall, a significant reduction from baseline to 6 months in problematic cannabis use was found using the CUDIT-R. This change was sustained at 12 months. Change in CUDIT-R score was strongly correlated with change in cannabis use diagnosis, and more modestly correlated with improvement in mood. Furthermore, using the CUDIT-R an expected association between motivation and change was demonstrated.

Thresholds for reliable change (2 or more points) and clinically significant change (3 or more points) have been identified. This establishes the CUDIT-R as a pragmatic measure that could be used in research and clinical settings. It is brief, covers a range of features of cannabis use beyond simple frequency, is freely available and straightforward to administer. For individual clinical work, simple self-report and measurement of urinary cannabinoids may be sufficient, but for systematic measurement in the absence of a budget for such an undertaking, simple pen and paper tools remain important.

For the purposes of the current analyses, cannabis users were divided into three categories based on their CUDIT-R score (Table 1). The lowest of these groups, termed "sub-threshold”, was for those scoring between 1 and 12, with a previous study showing 13 or more as the optimal cut-off for identifying a current cannabis use disorder diagnosis (Adamson et al., 2010). This should not be taken to mean that scores lower than 13 are not of clinical concern. A score of 12 would require very heavy use or somewhat heavy use and be accompanied by problems in at least one area. The score ranges adopted for these analyses were for exploratory purposes only and are not intended as a guide for interpretation of problem severity. This highlights the need for an analysis of CUDIT-R scores among community and clinical sample to develop interpretative norms. 
A one point change in criteria count for DSM-IV cannabis dependence was used as a benchmark for “clinical significance”. There is no simple, universally agreed measure of clinical significance, and so we have adopted this measure as something that might be meaningful in clinical practice. This also provides a useful benchmark for interpreting research findings using the CUDIT-R. In most cases, clinicians and their patients may be hoping for a more substantial change than a 3 point reduction in CUDIT-R score, but with this minimal level of meaningful change defined, it will be possible to be encouraged by smaller improvements, while being cautious to avoid over-interpreting reductions of 1 or 2 points. A reduction of 2 points was found to represent reliable change, that is, for an individual respondent a change of 2 points is likely to represent actual change in severity, rather than measurement error. This would appear to be of only slight clinical impact in and of itself.

Change in cannabis use was significantly associated with change in mood but not alcohol consumption, while the association between cannabis use and employment was weak and might be considered counterintuitive. Measures of cannabis use and depression are found to correlate in population surveys, with a modest causal association suggested by the data, primarly accounted for by outcomes for heavy cannabis users (Degenhardt et al. 2004; Moore et al. 2007). Changes in cannabis use were not associated with changes in alcohol use, suggesting that participants made different choices about the two substances, given that there were substantial reductions in use of both for participants in the SHADE trial (Kay-Lambkin et al., 2011). Finally, higher motivation to change cannabis use at baseline was found to predict reduction in CUDIT-R scores from baseline to 6 months. Motivation has been found to be a strong predictor of outcome for alcohol treatment (Adamson et al, 2009) but has been less studied in cannabis treatment.

Limitations of the study include the relatively modest sample size, significant loss to follow-up, a reliance on self-reported cannabis use, and the fact the primary study was not designed to test the 
properties of the CUDIT-R. The relatively small sample for the current analyses does increase the risk that a true effect does not reach significance, a false negative. Small sample size was compounded by a somewhat high loss to follow-up which occurred in the first six months with a relatively stable sample size thereafter. The reason for this high drop-out rate may in part be due to the relatively low motivation level reported for the sample. While this was a clinical sample, not everyone warranted intervention for their cannabis use, with a minority producing very low scores on the CUDIT-R at baseline. This is because the sample included not only those eligible for the study by virtue of their level of cannabis use, but also those eligible due to alcohol or amphetamine use who also reported some level of cannabis use. However we consider that this increased heterogeneity does improve the suitability of the sample to examine the clinical utility of the CUDIT-R for a variety of populations.

The CUDIT-R enquires about cannabis use and related experiences over the past 6 months. Although this will often be a suitable timeframe for research settings, ability to test more frequently would be helpful in routine clinical practice. It is not possible to shorten the timeframe to monthly as many of the items include response options of "less than monthly". However, modification of the CUDIT-R so that it could be re-administered after 3 months could be feasible and warrants further consideration.

\section{CONCLUSION}

As a brief 8-item assessment tool, the CUDIT-R provides a simple and reliable measure of the severity of problematic cannabis use. This paper extends the utility of the CUDIT-R by demonstrating its sensitivity to change in a treatment sample, with definitions of reliable and clinically significant change applicable to research and clinical settings.

\section{Declaration of interest}


Measuring Change in Cannabis Use

The authors report no conflict of interest. The authors alone are responsible for the content and writing of this article. The SHADE study was funded by the Alcohol Education Rehabilitation Foundation (AERF, Grant Number: AERFDOCS\2002\G0353). 


\section{REFERENCES}

Adamson, S.J., \& Sellman, J.D. (2003). A prototype screening instrument for cannabis use disorder: The Cannabis Use Disorders Identification Test (CUDIT) in an alcohol dependent clinical sample. Drug and Alcohol Review, 22, 309-315.

Adamson, S.J., Sellman, J.D., \& Frampton, C.M.A. (2009). Patient predictors of alcohol treatment outcome: A systematic review. Journal of Substance Abuse Treatment, 36, 75-86.

Adamson, S.J., Kay-Lambkin, F.J., Baker, A.L., Lewin, T.J., Thornton, L., Kelly, B.J., \& Sellman, J.D. (2010). An improved brief measure of cannabis misuse: The Cannabis Use Disorders Identification Test-Revised (CUDIT-R). Drug and Alcohol Dependence. 110, 137-143.

Annaheim, B., \& Scotto, T. J. (2010). Revising the Cannabis User Disorders Identification Test (CUDIT) by means of Item Response Theory. Journal of Methods in Psychiatric Research, 19(3), 142-155.

Annaheim B., Rehm J., \& Gmel G. (2008) How to screen for problematic cannabis use in population surveys. An evaluation of the Cannabis Use Disorders Identification Test (CUDIT) in a Swiss sample of adolescents and young adults. European Addiction Research, 14, 190-197,

Bashford, J., Flett, R., \& Copeland, J. (2010). The Cannabis Use Problems Identification Test (CUPIT): Development, reliability, concurrent and predictive validity among adolescents and adults. Addiction, 105, 615-625.

Beck, A.T. (1993). Beck Depression Inventory. San Antonio, Texas: Psychological Corporation. 
Measuring Change in Cannabis Use

Bolla, K.I., Brown, K., Eldreth, D., Tate, K., \& Cadet, J.L. (2002). Dose-related neurocognitive effects of marijuana use. Neurology, 59, 1337-1343.

Copeland, J., Swift, W., \& Rees, V. (2001). Clinical profile of participants in a brief intervention for cannabis use disorder. Journal of Substance. Abuse Treatment, 20, 45-52.

Degenhardt, L., Hall, W., \& Lynskey, M. (2003). Exploring the association between cannabis use and depression. Addiction, 98, 1493-1504.

Fergusson, D.M., Boden, J.M., \& Horwood, L.J. (2006). Cannabis use and other illicit drug use: Testing the cannabis gateway hypothesis. Addiction, 101, 556-569.

Fergusson, D. M., Horwood, L. J., \& Boden, J. M. (2008). Is driving under the influence of cannabis becoming a greater risk to driver safety than drink driving? Findings from a longitudinal study. Accident Analysis \& Prevention, 40, 1345-1350.

Gossop, M., Darke, S., Griffiths, P., Hando, J., Powis, B., Hall, W., \& Strang, J. (1995). The Severity of Dependence Scale (SDS): Psychometric properties of the SDS in English and Australian samples of heroin, cocaine and amphetamine users. Addiction, 90, 607-614.

Hall, W., \& Degenhardt, L. (2008). Cannabis use and the risk of developing a psychotic disorder. World Psychiatry, 7, 68-71.

Heather, N., Luce, A., Peck, D., Dunbar, B., \& James, I. (1999). Development of a treatment version of the Readiness to Change Questionnaire. Addiction Research, 7, 63-83. 
Heishman, S.J., Singleton, E.G. \& Ligouri, A. (2001). Marijuana Craving Questionnaire: Development and initial validation of a self-report instrument. Addiction, 96, 1023-1034.

Horwood, L.J., Fergusson, D.M., Hayatbakhsh, M.R., Najman, J.M., Coffey, C., Patton, G.C., Silins, E., \& Hutchinson, D.M. (2010). Cannabis use and educational achievement: findings from three Australasian cohort studies. Drug and Alcohol Dependence, 110, 247-253.

Jacabson, N.S., \& Truax, P. (1991). Clinical significance: a statistical approach to defining meaningful change in psychotherapy research. Journal of Consulting and Clinical Psychology, 59, 12-19.

Kay-Lambkin, F.J., Baker, A.L., Kelly, B., \& Lewin, T.J. (2011). Clinician-assisted computerised versus therapist-delivered treatment for depressive and addictive disorders: a randomised controlled trial. Medical Journal of Australia, 195, S44-S50.

McRae, A.L., Budney, A.J.,\& Brady, K.T. (2003). Treatment of marijuana dependence: a review of the literature. Journal of Substance Abuse Treatment, 24, 369-376.

Meier, M.H., Caspi, A., Ambler, A., Harrington, H., Houts, R., Keefe, R.S., McDonald, K., Ward, A., Poulton, R., \& Moffitt, T.E. (2012). Persistent cannabis users show neuropsychological decline from childhood to midlife. Proceedings of the National Academy of Science, 109, E2657-2664.

Moore, T.H.M., Zammit, S., Lingford-Hughes A., Barnes, T.R.E., Jones, P.B., Burke, M., \& Lewis, G. (2007). Cannabis use and risk of psychotic or affective mental health outcomes: a systematic review. Lancet, 370, 319-328. 
Measuring Change in Cannabis Use

Nordstrom, B.R., \& Levin, F.R. (2007). Treatment of cannabis use disorders: a review of the literature. American Journal on Addictions, 16, 331-342.

Patton, G.C., Coffey, C., Carlin, J.B., Degenhardt, L., Lynskey, M., \& Hall, W. (2002). Cannabis use and mental health in young people: cohort study. British Medical Journal, 325, 1195-1198.

Pope, H,G. Jr., Gruber, A.J., Hudson, J.I, Huestis, M.A., \& Yurgelun-Todd, D. (2001) Neuropsychological performance in long-term cannabis users. Archives of General Psychiatry, 58, 909-15.

Piontek D., Kraus L., Klempova D. (2008) Short scales to assess cannabis-related problems: A review of psychometric properties. Substance Abuse Treatment, Prevention, and Policy, 3, 25,

Ramaekers, J. G., Berghaus, G., van Laar, M., \& Drummer, O. H. (2004). Dose related risk of motor vehicle crashes after cannabis use. Drug and Alcohol Dependence, 73, 109-119.

Saunders, J.B., Aasland, O.G., Babor, T.F., de la Fuente, J.R., \& Grant, M. (1993). Development of the Alcohol Use Disorders Identification Test (AUDIT): WHO Collaborative Project on Early Detection of Persons with Harmful Alcohol Consumption-II. Addiction, 88, 791-804.

Spitzer, R.L., Williams, J.B.W., Gibbon, M., \& First, M.B. (1988). Structured Clinical Interview for DSM-III-R-Patient Version (SCID-P). Biometrics Research Department, New York State Psychiatric Institute, New York. 
Measuring Change in Cannabis Use

Tashkin, D. P. (1999). Effects of cannabis on the respiratory system. In: H. Kalant, W. Corrigall, W. D. Hall, \& R. Smart (Eds.), The Health Effects of Cannabis (pp. 311-345). Toronto: Centre for Addiction and Mental Health.

United Nations Office on Drugs and Crime, (2010). 2010 World Drug Report: United Nations Publication Sales No. E. 10.XI.5 (ISBN 978-92-1-148256-0).

WHO ASSIST Working Group, (2002). The Alcohol, Smoking and Substance Involvement Screening Test (ASSIST): Development, reliability and feasibility. Addiction, 97, 1183-1194. 
Measuring Change in Cannabis Use

Table 1: Distribution of CUDIT-R scores at baseline and follow-up

\begin{tabular}{|c|c|c|c|c|c|c|}
\hline \multirow[b]{2}{*}{ Mean } & \multicolumn{2}{|c|}{ Baseline $(n=129)$} & \multicolumn{2}{|c|}{6 Months $(n=80)$} & \multicolumn{2}{|c|}{12 Months $(n=78)$} \\
\hline & 18.37 & & 13.44 & & 12.60 & \\
\hline SD & 9.89 & & 11.30 & & 11.55 & \\
\hline SE & 0.871 & & 1.26 & & 1.308 & \\
\hline \multicolumn{7}{|c|}{ Cannabis problem level } \\
\hline by CUDIT-R Score & $n$ & $\%$ & $n$ & $\%$ & $n$ & $\%$ \\
\hline No use (0) & 0 & 0 & 20 & 25.0 & 25 & 32.1 \\
\hline Sub-threshold (1-12) & 37 & 28.7 & 19 & 23.8 & 18 & 23.1 \\
\hline Medium (13-20) & 32 & 24.8 & 16 & 20.0 & 10 & 12.8 \\
\hline High (21-32) & 60 & 46.5 & 25 & 31.25 & 25 & 32.1 \\
\hline
\end{tabular}


Measuring Change in Cannabis Use

Table 2: Mean change and application of the Reliable Change Index

Baseline to $12 \quad 6$ months to 12

\begin{tabular}{|c|c|c|c|c|}
\hline & Baseline to 6 months & months & months & 1 week test-retest \\
\hline $\mathrm{N}$ & 76 & 74 & 64 & 66 \\
\hline Mean change & -5.49 & -6.50 & -0.61 & -0.44 \\
\hline SD change & 10.69 & 10.68 & 5.40 & 4.47 \\
\hline \% improved & $65.8 \%$ & $67.6 \%$ & $35.9 \%$ & $40.9 \%$ \\
\hline \%unchanged ( \pm 1$)$ & $15.8 \%^{1}$ & $17.6 \%^{1}$ & $40.6 \%$ & $39.9 \%{ }^{1}$ \\
\hline$\%$ deteriorated & $18.4 \%$ & $14.9 \%$ & $23.4 \%$ & $19.7 \%$ \\
\hline
\end{tabular}

${ }^{1}$ Note that $3 \%$ of the sample scored 1 at baseline and so were incapable of being categorised as improved, even if they became abstinent. 
Measuring Change in Cannabis Use

Table 3: Change in cannabis diagnosis from baseline to 6 months

6 months diagnostic category, $\mathrm{n}=77$

\begin{tabular}{|c|c|c|c|c|c|}
\hline Baseline & Abstinent & Cannabis use, no & Cannabis Abuse & Cannabis & Total \\
\hline Diagnostic & & diagnosis & & Dependence & \\
\hline
\end{tabular}

category

Cannabis use, no

$\begin{array}{cc}5 & 9 \\ & \\ 1 & 6 \\ 10 & 10\end{array}$

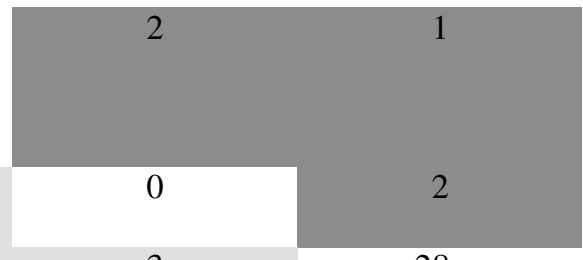

17

diagnosis

Cannabis Abuse

10

3

28

9

Cannabis

Dependence

$$
\text { Improved = 35(45.5\%) }
$$

Unchanged

$$
=37(48.1 \%)
$$


Measuring Change in Cannabis Use

\begin{tabular}{|c|c|c|c|c|c|c|c|c|c|}
\hline & \multicolumn{3}{|c|}{ Baseline to 6 months } & \multicolumn{3}{|c|}{ Baseline to 12 months } & \multicolumn{3}{|c|}{6 months to 12 months } \\
\hline & $\mathrm{N}$ & $r$ & $p$ & $\mathrm{~N}$ & $r$ & $p$ & $\mathrm{~N}$ & rho & $P$ \\
\hline BDI-II & 75 & 0.230 & 0.047 & 73 & 0.301 & 0.010 & 64 & 0.249 & 0.047 \\
\hline \multirow[t]{2}{*}{ AUDIT } & 69 & 0.105 & 0.389 & 66 & 0.182 & 0.144 & 59 & -0.187 & 0.155 \\
\hline & & rho & & & rho & & & rho & \\
\hline Employment & 76 & -0.137 & 0.236 & 72 & -0.218 & 0.066 & 62 & 0.250 & 0.050 \\
\hline
\end{tabular}

Note: BDI-II: Beck Depression Inventory-II (Beck, 1993); AUDIT: Alcohol User Disorders Identification Test (Saunders et al., 1993) 


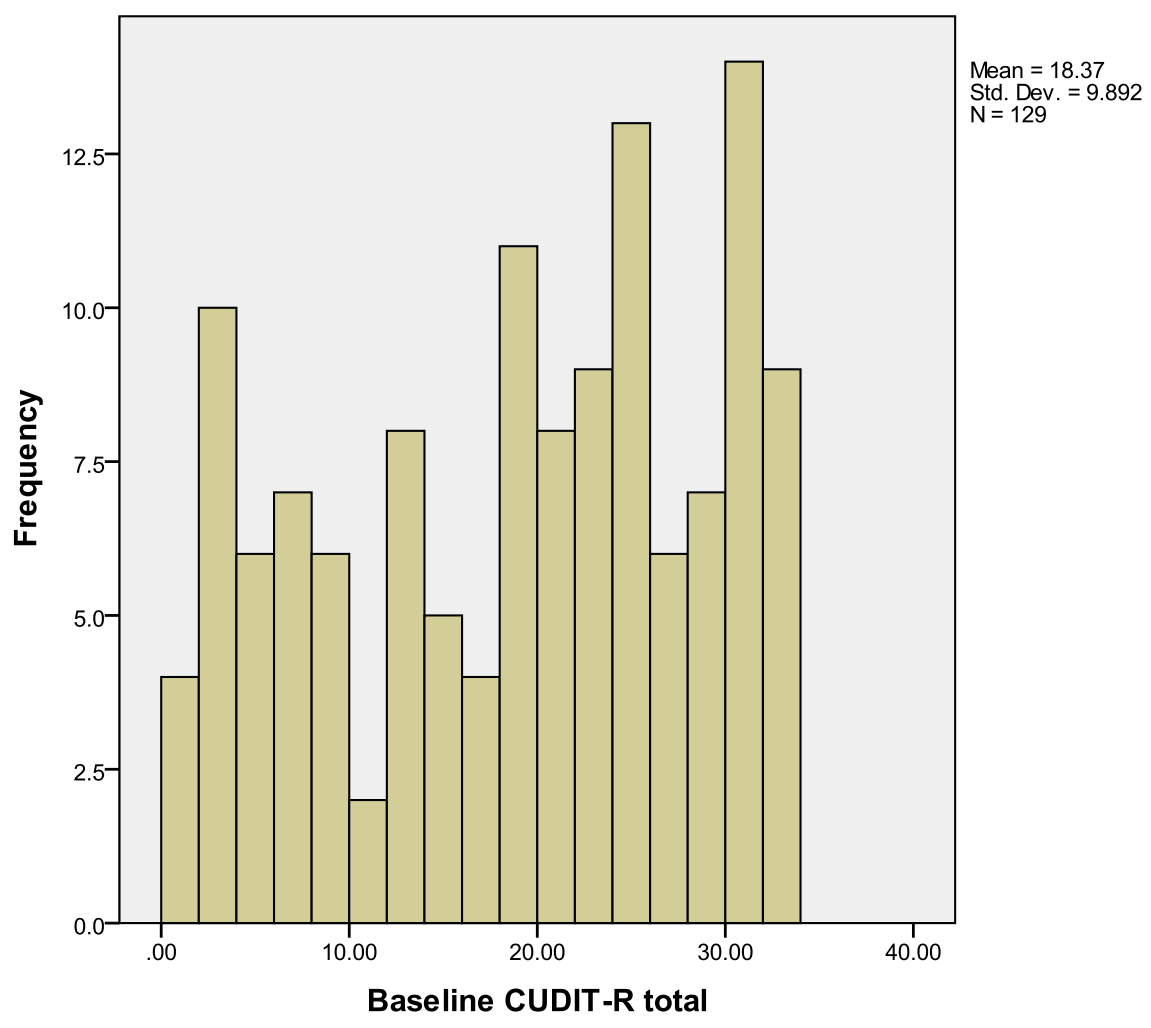

Figure 1: Distribution of baseline CUDIT-R scores 


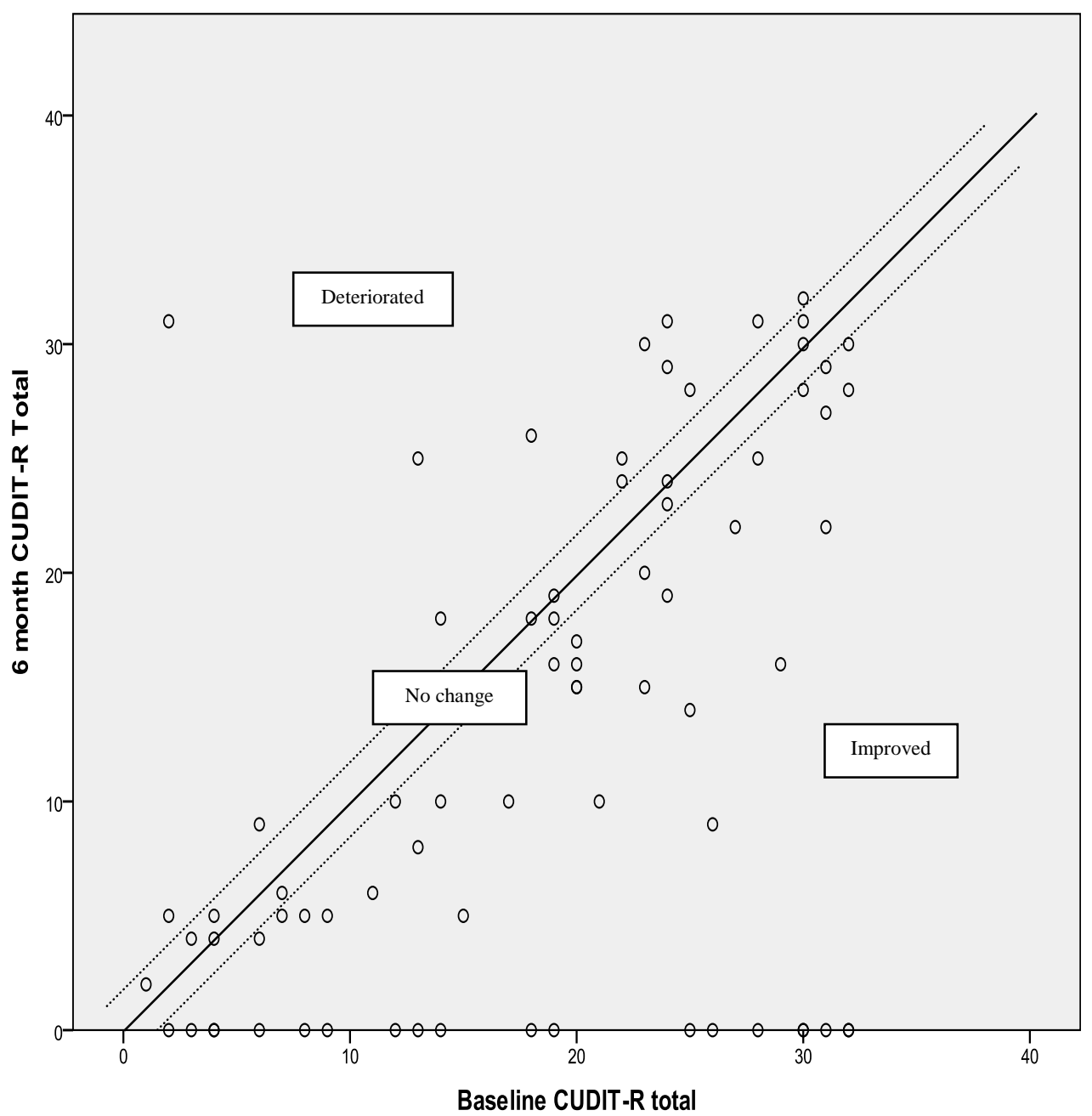

Note: Parallel dotted lines represent the boundary between no reliable change $( \pm 1)$ and improvement/deterioration $( \pm 2$ or more)

Figure 2: CUDIT-R scores, change from baseline to 6 months, n=76 